\title{
Estimating reflectivity values from wind turbines for analyzing the potential impact on weather radar services
}

\author{
I. Angulo ${ }^{1}$, O. Grande ${ }^{1}$, D. Jenn ${ }^{2}$, D. Guerra ${ }^{1}$, and D. de la Vega ${ }^{1}$ \\ ${ }^{1}$ University of the Basque Country (UPV/EHU), Bilbao, Spain \\ ${ }^{2}$ Naval Postgraduate School, Monterey, USA \\ Correspondence to: D. de la Vega (david.delavega@ehu.es) \\ Received: 16 January 2015 - Published in Atmos. Meas. Tech. Discuss.: 3 February 2015 \\ Revised: 28 April 2015 - Accepted: 30 April 2015 - Published: 27 May 2015
}

\begin{abstract}
The World Meteorological Organization (WMO) has repeatedly expressed concern over the increasing number of impact cases of wind turbine farms on weather radars. Current signal processing techniques to mitigate wind turbine clutter (WTC) are scarce, so the most practical approach to this issue is the assessment of the potential interference from a wind farm before it is installed. To do so, and in order to obtain a WTC reflectivity model, it is crucial to estimate the radar cross section (RCS) of the wind turbines to be built, which represents the power percentage of the radar signal that is backscattered to the radar receiver.

For the proposed model, a representative scenario has been chosen in which both the weather radar and the wind farm are placed on clear areas; i.e., wind turbines are supposed to be illuminated only by the lowest elevation angles of the radar beam.

This paper first characterizes the RCS of wind turbines in the weather radar frequency bands by means of computer simulations based on the physical optics theory and then proposes a simplified model to estimate wind turbine RCS values. This model is of great help in the evaluation of the potential impact of a certain wind farm on the weather radar operation.
\end{abstract}

\section{Introduction}

The potential impact of wind turbines on weather radar performance has been extensively studied in the last few years, with several evidences of wind turbine clutter observations in meteorological radar applications (Isom et al., 2008; Gallardo-Hernando et al., 2011; Norin and Haase, 2012; Vogt et al., 2011; WMO, 2005, 2010). The main objective of these studies is to characterize and try to mitigate the so-called wind turbine clutter (WTC), mainly by means of digital signal processing such as clutter-filtering techniques.

Unfortunately, these solutions are not widely available yet. Meanwhile, the most practical approach to this issue is the prediction of the potential impact on a certain weather radar service before installing a wind farm. In most cases, the identification of a potential impact allows the planning of alternative solutions in order to guarantee the coexistence of wind energy and meteorological radar services.

Wind turbine clutter reflectivity depends on many factors including wind turbine dimensions, wind direction and velocity, angle of incidence and radar frequency (GallardoHernando et al., 2011; Norin and Haase, 2012; Norin, 2015). In order to measure how efficiently radar pulses are backscattered by wind turbines, existing models of wind turbine clutter and weather radar recommendations rely on the turbines' radar cross section (RCS) (Tristant, 2006; ITU-R, 2009; Norin and Haase, 2012). The RCS is the projected area required to intercept and isotropically radiate the same power as the target scatters toward the receiver, and thus it is normally expressed in decibels with respect to a square meter (dBsm) (Skolnik, 2008; Rinehart, 1997).

In this context, the goal of this paper is to propose simplified formulae for the estimation of reflectivity values from wind turbines at frequencies used by weather radars. These formulae aim at being easily implementable in software tools for estimating the potential impact of wind farms on weather radars. 
For this purpose, first RCS patterns for different working conditions of the wind turbines are obtained by means of physical optics (PO) simulations and subsequently analyzed. Additionally, separate RCS patterns of the parts of the turbine are also calculated in order to compare the relative contribution of each component. Based on these simulations, a simple algorithm to evaluate the potential impact of a wind farm on a nearby weather radar is proposed.

It should be mentioned that similar studies for characterizing RCS of wind turbines have been carried out for evaluating the impact on different services such as maritime radars (Grande et al., 2014) or television (Angulo et al., 2011). However, as scattering is very dependent on working frequency and illumination conditions, results cannot be extrapolated. Moreover, preliminary results of the analysis presented in this paper are included in a previous communication from the authors (Grande et al., 2015). Those results correspond to a single wind turbine model and a single working frequency. In the present paper, results are extended to three wind turbine models of different size and the three frequency bands assigned to weather radar services; besides, based on the obtained results, a novel formulation for estimating the WTC reflectivity values for weather radar applications is proposed. This work aims at making studies for the prediction of potential impacts between weather radar services and wind farm deployments easier to conduct.

\section{Impact of wind farms on weather radars}

In weather radars, wind turbines may lead to misidentification of precipitation features and to erroneous characterization of meteorological phenomena. These errors may be due to clutter caused by signal echoes from the wind turbines; signal blockage, as the physical size of the wind turbine creates a shadow zone behind them of diminished detection capacity; and interference to the Doppler mode of the radar, on account of frequency shifted echoes from the rotating blades (Angulo et al., 2014; Norin and Haase, 2012; Belmonte and Fabregas, 2010).

The clutter from wind turbines is due to radar echoes coming from a turbine and reaching the radar with a power level higher than the radar detection threshold, preventing the radar from correctly detecting the precipitation level in the affected area. Although most of current radars include signal processing techniques that remove static scattering from turbine masts, the scattered energy will increase the effective noise floor of the radar receiver, which degrades the detection capacity and therefore the data quality obtained by the radar. Detection of precipitation requires a signal that exceeds the noise floor by at least the signal-to-noise ratio. Energy scattered from wind turbines results in the occurrence of increased noise that might cause desired targets to be undetected. Although the signal processing techniques may mitigate the display of false targets generated by the stationary clutter from a wind farm, it will not eliminate effects that raise the noise floor of the radar (Tristant, 2006; Lemmon et al., 2008).

Regarding the Doppler mode of the radar, as it is aimed at detecting moving targets, only the scattering from the blades should be considered in order to determine the influence of a wind turbine on this operation mode.

Therefore, both the clutter phenomenon and the interference to the Doppler mode depend on the scattering characteristics of wind turbines. By contrast, as the blocking of the radar beam is due to the physical obstruction of the radar beam by the wind turbine, the methodology to estimate a potential impact of a wind farm due to signal blockage is not related to the RCS of wind turbines but to the percentage of the beam section blocked by the wind turbine structure (Tristant, 2006; Belmonte and Fabregas, 2010). Consequently, this paper does not focus on addressing the signal blockage estimates.

As the RCS of a wind turbine depends both on fixed parameters, such as the dimensions and materials of each component of the wind turbine, and on variable parameters, such as position of the rotating blades and rotor orientation with respect to the radar, RCS values may vary drastically according to wind turbine working regimes and illumination conditions (Angulo et al., 2011; Grande et al., 2014).

The calculation of RCS values by conventional prediction methods, such as the method of moments (MoM) or the finite difference time domain (FDTD) method, provides accurate results but relies upon extremely detailed representations of the turbine, which requires significant modeling and complex calculations with great computational effort. Consequently, these RCS prediction methods cannot be easily implemented in computer simulation tools for analyzing the potential impact of a specific wind farm.

On the contrary, and due to the absence of simplified formulation, some published guidelines for analyzing the impact of wind turbines on radar services use typical fixed RCS values, disregarding the particular features of each installation (ITU-R, 2009; Tristant, 2006). This is a very simple way to deal with wind turbine scattering, but its main disadvantage is that the proposed RCS values do not take into account the characteristics of the real scenario under analysis: wind turbine dimensions, angle of incidence and working frequency, amongst others. As a result, these proposed typical constant RCS values may lead to important estimation errors.

In this paper, a simplified formulation for determining accurate WTC reflectivity values is proposed. The presented method requires neither complex calculations nor the use of a simulation tool, whereas it provides RCS values adapted to the particular features of the case under analysis: dimensions of the wind turbine models, illumination conditions and working frequency. 


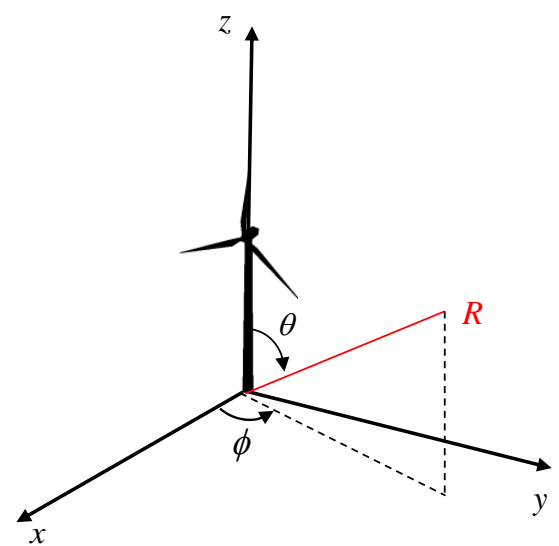

Figure 1. Spherical coordinate system used in the RCS calculations. $R$ represents radar location.

\section{Methodology}

The main objective of this paper is to develop an estimation model of wind turbine reflectivity values for weather radars that consists in a simplified formulation, is easy to apply in the development of the impact studies, and does not require a complex software tool or a high amount of resources.

The estimation model should fulfill the following conditions.

- Despite being a simplified formulation, the model should provide accurate radar cross-section values, which are directly translated into reflectivity values.

- The model should consider the variability of the RCS values generated by the rotor orientation and the blades rotation, because the RCS values are very dependent on the specific relative positions of the different components of the turbine with respect to the radar.

- The model should be applicable to turbine models of different size, different working frequencies and different radar illumination conditions.

\subsection{Simulation conditions}

\subsubsection{Simulation tool and wind turbine models}

The present study is based on the accurate assessment of RCS values of wind turbines by applying the PO theory. The PO theory is a high-frequency approximation method that provides accurate results for electrically large objects $(L \geq 10 \lambda)$ and for observation points near the specular direction. More precisely, the software tool POfacets (Jenn, 2005) has been used to calculate RCS patterns of three different wind turbine models. To do so, detailed facet-based representations of these wind turbine models have been prepared for the application of numerical solutions of the PO method for RCS estimations. The software tool does not include the effect of
Table 1. Wind turbine models selected for the simulations.

\begin{tabular}{llll}
\hline & Model 1 & Model 2 & Model 3 \\
\hline Mast height & $78 \mathrm{~m}$ & $100 \mathrm{~m}$ & $119 \mathrm{~m}$ \\
Mast upper radius & $1.15 \mathrm{~m}$ & $1.80 \mathrm{~m}$ & $2.40 \mathrm{~m}$ \\
Mast lower radius & $1.75 \mathrm{~m}$ & $2.70 \mathrm{~m}$ & $3.60 \mathrm{~m}$ \\
Rotor diameter & $87 \mathrm{~m}$ & $90 \mathrm{~m}$ & $112 \mathrm{~m}$ \\
Blade length & $42.50 \mathrm{~m}$ & $44.00 \mathrm{~m}$ & $54.65 \mathrm{~m}$ \\
Rated power & $2.0 \mathrm{MW}$ & $2.0 \mathrm{MW}$ & $3.3 \mathrm{MW}$ \\
\hline
\end{tabular}

multiple reflections, diffraction or surface waves. More in depth descriptions of the physical optics method and the simulation tool can be found in Jenn (2005), Grande et al. (2014, 2015).

It should be noted that this tool provides accurate RCS values for a specific rotor orientation and blade position but at the expense of having to design rigorous representations of the wind turbine models. Hence, estimations of RCS values for each specific position of the blades must be conducted and therefore hundreds of RCS simulations are required in order to obtain a detailed characterization of the RCS patterns for different working conditions. The analysis of this huge set of RCS values is the basis of the proposed simplified model to be integrated in the prediction tools for potential interference from a wind farm. In fact, the main motivation of the proposed simplified model is precisely avoiding the need of such a simulation effort in future cases under study.

As mentioned in Sect. 1, three commercial wind turbine models were chosen for the analysis, which constitutes a representative selection of the wind turbines that are usually installed. Typical horizontal-axis wind turbines are composed of a mast or supporting tower, commonly made from tubular steel; a nacelle that holds all the turbine machinery and rotates to follow the wind direction; and a rotor with three blades of complex aerodynamic surface, being the rotor shaft tilted above the horizontal to enable greater clearance between the blades and the mast. Characteristics of the selected models are summarized in Table 1. It should be noted that upper and lower radii of the masts are different because the geometry of the supporting tower of the wind turbines is not a perfect right circular cylinder but a tapered cylinder.

Figure 1 shows the reference coordinate system for the analysis. The wind turbine rotor is supposed to be oriented towards the $x$ axis and $R$ refers to the radar position. As shown in the figure, $\theta$ is the angle from the zenith that defines the radar position in the vertical plane, and $\phi$ specifies the horizontal position of the radar with respect to the rotor orientation, i.e., with respect to the rotor shaft axis.

\subsubsection{Simulation precision}

The analysis is based on the assessment of backscattering patterns for a set of elevation angles (variation in $\theta$ ), as detailed in Sect. 3.2, and different conditions of rotor orienta- 


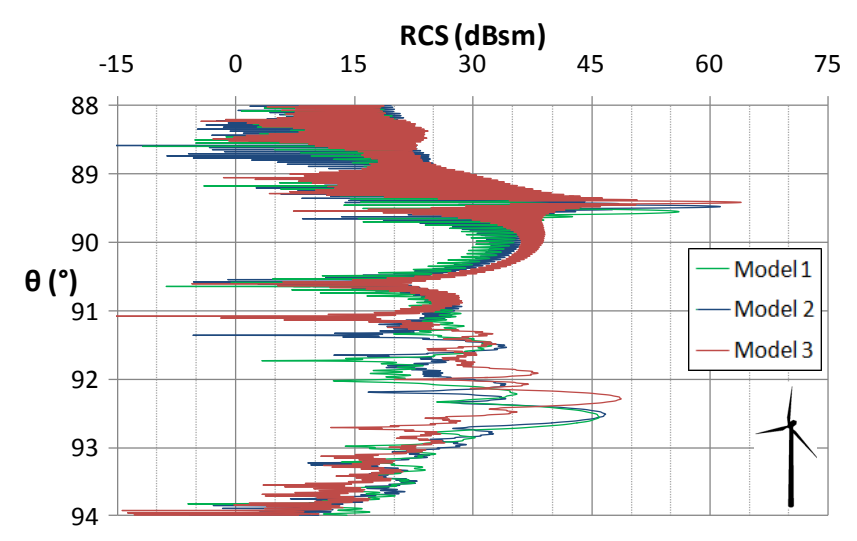

Figure 2. Vertical sections of RCS patterns $\left(\phi=5^{\circ}\right)$ for wind turbine models 1 to 3 at frequency $2.80 \mathrm{GHz}$. Rotor position is indicated in the lowest right corner.

tion with respect to the radar (variation in $\phi$ from 0 to $185^{\circ}$ ) and blades position (rotating blades).

Calculations with particularly high resolution have been conducted for RCS vertical patterns (resolution of $0.001^{\circ}$ in $\theta$ ), as great variability is expected in this plane. The effect of the rotating blades has been analyzed by simulations with a difference of $15^{\circ}$ in the rotation angle of the blades. In addition, these estimated RCS values have been obtained for different positions of the rotor with respect to the incident signal in the horizontal plane (aspect angles separated $1^{\circ}$ in $\phi)$.

In order to evaluate the relative significance of the signal backscattered by the different parts of the wind turbine, separated RCS patterns of the mast, nacelle and single blades have been obtained and compared with the RCS pattern of the whole wind turbine, as described in Sect. 4 and shown in Figs. 2 to 7.

\subsection{Considerations of the analysis}

The case under analysis is a wind farm located within the detection volume of a weather radar. When this situation occurs, some specific conditions are applicable. The thorough outline of these conditions allows the clear delimitation of the scenario under analysis:

- Monostatic backscattering. Weather radars only receive monostatic backscattered signals, so monostatic RCS values are analyzed in this paper.

- Frequency bands. The analysis is conducted for the frequency bands assigned to weather radar operation: $2700-2900 \mathrm{MHz}$ in $\mathrm{S}$ band, $5250-5725 \mathrm{MHz}$ (mainly $5600-5650 \mathrm{MHz}$ ) in $\mathrm{C}$ band and $9300-9500 \mathrm{GHz}$ in $\mathrm{X}$ band (ITU-R, 2008). In weather radars, $\mathrm{S}$ band is well suited for detecting heavy rain at very long ranges, up to $300 \mathrm{~km} ; \mathrm{C}$ band represents a good compromise between range and reflectivity and cost, and they can provide rain detection up to $200 \mathrm{~km}$; and $\mathrm{X}$ band weather radars are used only for short range weather observations up to a range of $50 \mathrm{~km}$ (ITU-R and WMO, 2008).

- Materials. The metallic mast can be considered as perfect electric conductor. Although modern blades are made of composite materials which are difficult to characterize, blades in the simulations are supposed to be metallic in order to consider the worst-case assumption for this component of the turbine.

- Relative location of weather radar and wind turbine, and corresponding elevation angles. As a proof of concept for the proposed model, a representative scenario has been chosen. This scenario considers that weather radars are usually located in open places that allow unobstructed scanning of a wide area, up to $300 \mathrm{~km}$. Wind farms are also placed on clear areas, where potential wind energy is higher. As weather radar beams use quite directive lobes (usually $1^{\circ}$ beam width), wind turbines are illuminated only when radar transmission is pointing to the wind farm. Therefore, the scenario that must be analyzed is the potential incidence of the lowest elevation angles of the radar beam on the wind turbines. Lowest elevation angles of the scanning routine are usually transmitted just above horizon for radar located in flat areas or slightly below the horizon for radars located on top of the hills. Accordingly, a reasonable range of the lowest elevation angles where the radar beam can illuminate a wind turbine is -2 to $+4^{\circ}$ with respect to the horizon (WMO, 2014) (Grande et al., 2015). The previous assumption leads to incidence angles on the wind turbine nearly perpendicular to the vertical axis of the mast, in particular within the range $88^{\circ}<\theta<94^{\circ}$.

- Reflectivity model. The calculation of the reflectivity value from a wind turbine is based on considering line of sight (LoS) propagation. In real scenarios, interactions from the ground and terrain should be taken into account, e.g., potential shadowing effects (Norin and Haase, 2012). Moreover, it is assumed that the wind turbine is being illuminated by the main lobe of the radiation pattern of the radar.

\section{Simulation results and analysis}

As mentioned in Sect. 3.2, simulations have been carried out for three frequencies representative of the different weather radar frequency bands $(2.80,5.65$ and $9.40 \mathrm{GHz})$ and three wind turbine models based on actual commercial turbines.

As an example, Figs. 2 to 4 show the vertical variation of the RCS patterns of wind turbine models 1 to 3 for a specific rotor orientation for the three frequencies under analysis. It can be observed that the RCS patterns show great variability and a very directive main lobe is noticeable in all cases. 


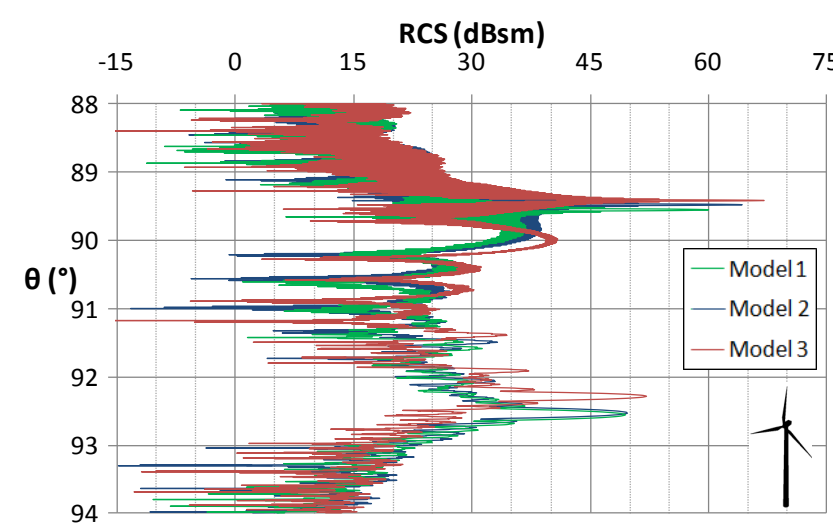

Figure 3. Vertical sections of RCS patterns $\left(\phi=5^{\circ}\right)$ for wind turbine models 1 to 3 at frequency $5.65 \mathrm{GHz}$. Rotor position is indicated in the lowest right corner.

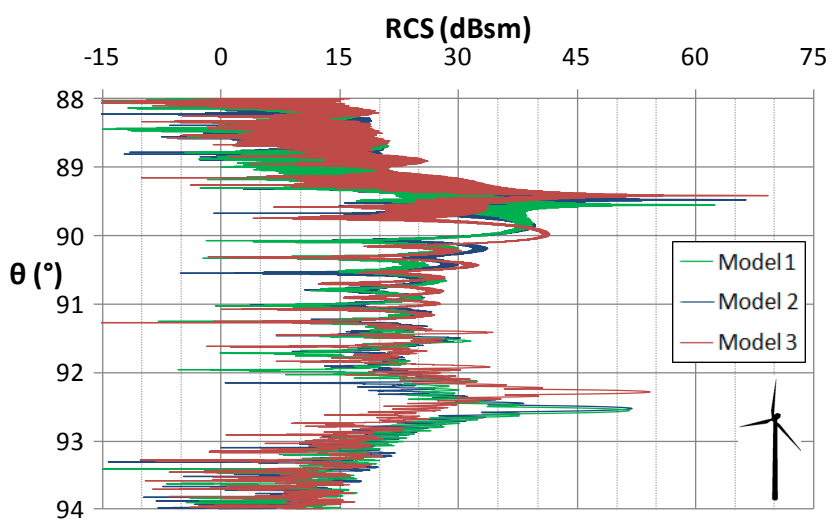

Figure 4. Vertical sections of RCS patterns $\left(\phi=5^{\circ}\right)$ for wind turbine models 1 to 3 at frequency $9.40 \mathrm{GHz}$. Rotor position is indicated in the lowest right corner.

This maximum value of the RCS corresponds to an illumination direction of $\theta=89.56^{\circ}$ with respect to the zenith in case of WT model $1, \theta=89.48^{\circ}$ in case of WT model 2 , and $\theta=89.42^{\circ}$ for WT model 3. Taking into account the slant surface of the masts, these directions correspond to the direction normal to the mast surface of each wind turbine model. As expected, the maximum RCS value is larger for the tallest wind turbine. Moreover, when comparing Fig. 2 to Fig. 4, it is clearly observed that the main lobe is both higher and narrower as the frequency increases. This maximum value of the RCS in the vertical pattern is maintained for all the azimuth values due to the symmetry of the mast in the horizontal plane.

In order to identify the contribution of the blades and nacelle, for the highest frequency and a specific rotor orientation, the RCS of WT model 3 is depicted in Fig. 5 for different positions of the blades (every $30^{\circ}$ in the rotation movement). The RCS pattern of the isolated mast is also depicted in Fig. 5. As observed in the figure, whereas the contribution

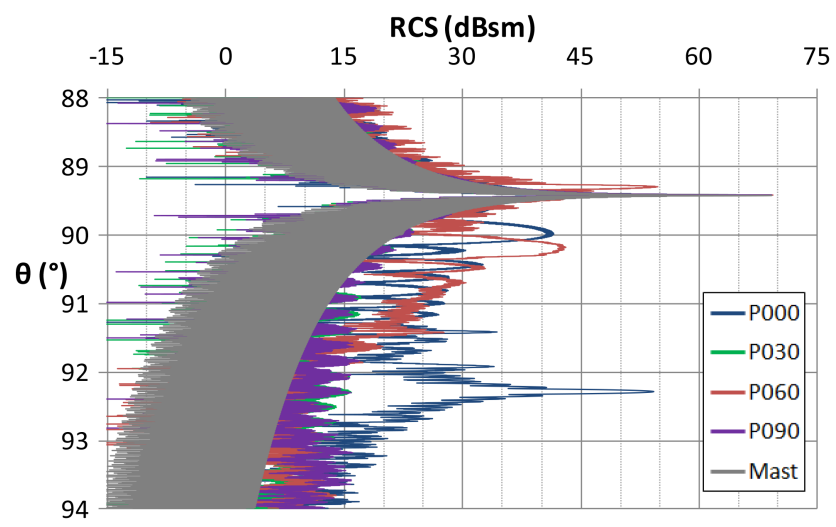

Figure 5. Vertical sections of RCS patterns $\left(\phi=5^{\circ}\right)$ for wind turbine model 3 at frequency $9.40 \mathrm{GHz}$. Legend entries starting with PXXX indicate the position of the upper blade (being P000 vertical right position and $\mathrm{P} 090$ horizontal position).

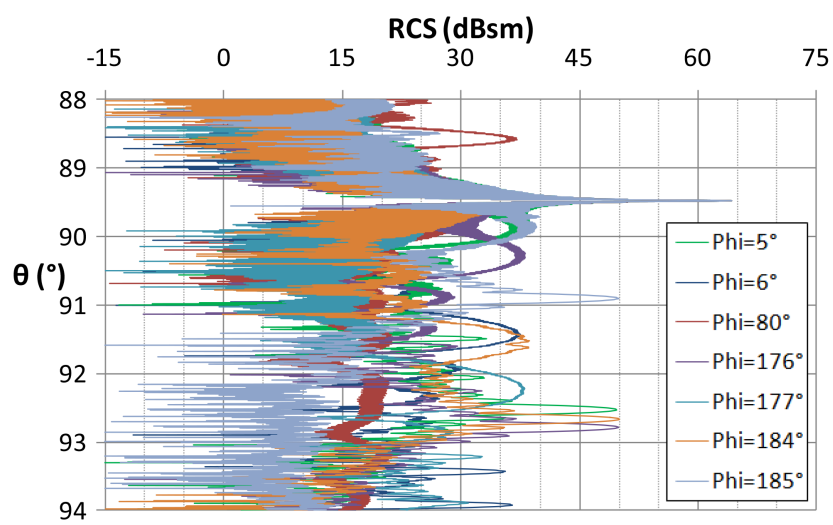

Figure 6. Vertical sections of RCS patterns ( $\phi=$ $5^{\circ}, 6,80,176,177,184,185^{\circ}$ ) for wind turbine model 2 at frequency $5.65 \mathrm{GHz}$ and rotor position $\mathrm{P} 000$.

from the blades varies in amplitude and position with the rotation movement, the maximum RCS of the wind turbine is constant and it is clearly generated by the mast. Figure 5 also shows that the main contribution from the rotor is due to a blade being in vertical position (see curves related to P000 and P060 in Fig. 5).

As it can be expected, the contribution from the blades is strongly dependent on the rotor orientation with respect to the incident radar signal, whereas the contribution from the mast remains invariable in the horizontal plane due to its symmetry with respect to the vertical axis of the mast. This statement is confirmed by Fig. 6, in which the vertical RCS patterns of WT model 2 are compared for different illumination directions in the horizontal plane (different $\phi$ values).

A first important conclusion obtained from the extensive set of simulations carried out is that the main scatterer of the wind turbine for the different frequency bands used for weather radar is the supporting mast. Moreover, the main feature of the scattering pattern of the mast is a main lobe 


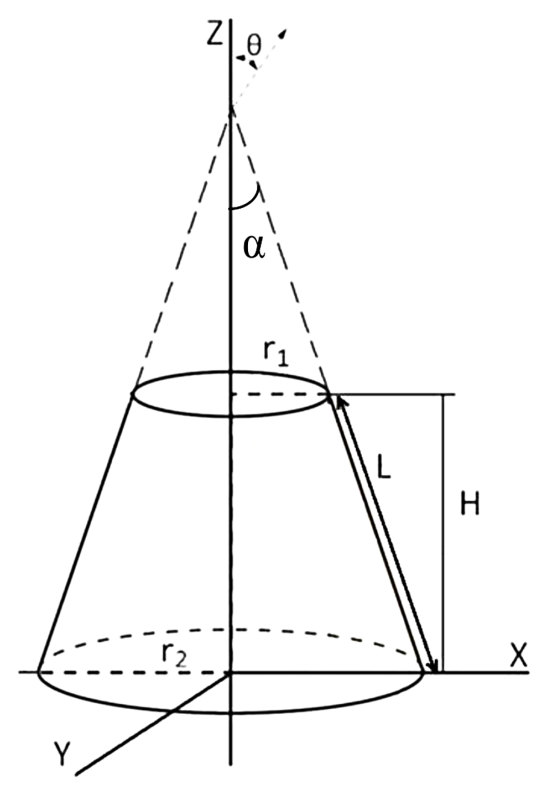

Figure 7. Geometry for the RCS calculation of the mast.

normal to the slant surface, extremely directive in the vertical plane and omnidirectional in the horizontal plane. The scattering from the mast can be approximated by the RCS of a right circular cylinder, which will be the basis of the proposed model for calculating the wind turbine RCS values, as later described in Sect. 5.1.

The blades, by contrast, provide variable levels of signal scattering depending on the rotor orientation and blade positions. Despite the variability of the scattering from the blades, their contribution to the total RCS of the wind turbine is always significantly lower than the amplitude of the main lobe due to the mast. Therefore, in order to provide a worst-case assumption with respect to the signal scattered by the blades, the proposed scattering model will provide an upper limit to the RCS values from the blades, as will be shown in Sect. 5.2.

\section{Proposed model}

\subsection{Scattering from the mast}

As demonstrated in the previous section, the mast is the main scatterer of the wind turbine due to its large dimensions, as it generates the maximum value of the RCS pattern.

The geometry of the mast can be approximated by a right cylinder because for commercial wind turbine models the half cone angle $\alpha$ that defines the slant surface of the mast is small (see Fig. 7):

$\alpha=\tan ^{-1}\left(\frac{r_{2}-r_{1}}{H}\right)$.

For example, for the three models under analysis, the half cone angle is smaller than $0.6^{\circ}$. Therefore, a perfectly con-

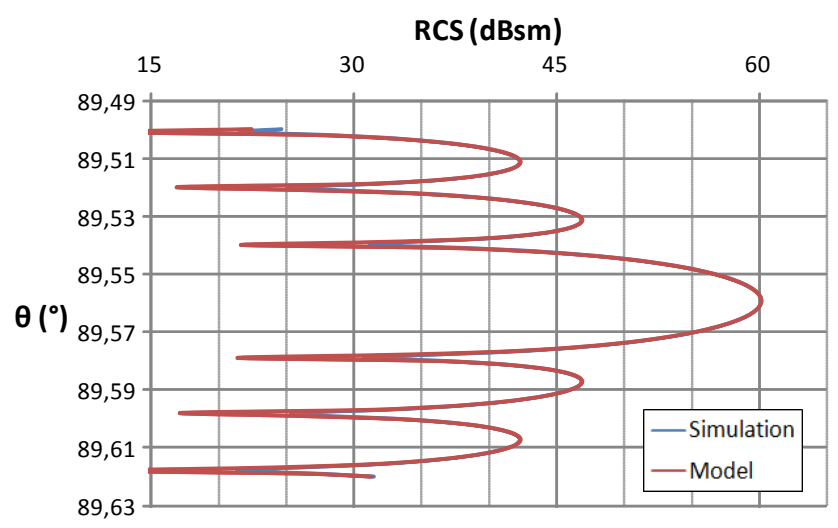

Figure 8. RCS pattern obtained by simulation vs. RCS values obtained by the proposed simplified model for the mast of wind turbine model 1 and frequency $5.65 \mathrm{GHz}$.

ducting right cylinder tilted at an angle $\alpha$ is used to assess the backscattered RCS of the mast based on the PO theory.

In Siegel et al. (1955) the RCS pattern of an elliptic cylinder is obtained as a function of its dimensions and the angular positions of the transmitter and receiver in both the vertical and the horizontal planes. The expression proposed in (Siegel et al., 1995) was adapted to a circular cylinder and simplified to avoid indeterminate forms as described in Appendix A of Angulo et al. (2013). As for radar applications only backscattering is of interest, the formulae in Angulo et al. (2013) for a circular cylinder can be further simplified assuming that $\theta_{t}=\theta_{r}$ and $\phi=0^{\circ}$ and expressed as

$\sigma_{\text {cylinder }}=\frac{2 \pi}{\lambda} r L^{2} \sin \theta\left(\frac{\sin \left(\frac{2 \pi}{\lambda} L \cos \theta\right)}{\frac{2 \pi}{\lambda} L \cos \theta}\right)^{2}$,

where $\lambda$ is the wavelength of the radar transmission, $\theta$ is the aspect angle as defined in Fig. 7, $r$ is the cylinder radius and $L$ is the cylinder height.

In order to adapt the previous expression to the actual geometry of the mast, two approximations are considered.

1. In (Skolnik, 2008), it is stated that Eq. (2) may be used to estimate the RCS of a truncated right circular cone if the radius $r$ is replaced by the mean radius of the cone and $L$ is replaced by the length of the slanted surface.

2. Taking into account the results of the previous section, it is clear that the backscattering pattern of the mast is extremely directive in the direction perpendicular to the slanted surface of the mast. Therefore, Eq. (2) should be slightly modified in order to account for the half cone angle $\alpha$. 
According to the above-mentioned considerations, the proposed model to calculate the RCS of the wind turbine mast is given by

$\sigma_{\text {mast }}=\frac{2 \pi}{\lambda} r L^{2} \sin (\theta+\alpha)\left(\frac{\sin \left(\frac{2 \pi}{\lambda} L \cos (\theta+\alpha)\right)}{\frac{2 \pi}{\lambda} L \cos (\theta+\alpha)}\right)^{2}$,

where $\lambda$ is the wavelength of the radar transmission, $\theta$ is the aspect angle as defined in Fig. 7, $\alpha$ is the half cone angle as given by Eq. (1), $r$ is the mean radius of the truncated cone

$r=\frac{r_{1}+r_{2}}{2}$,

and $L$ is the length of the slanted surface of the mast

$L=\frac{H}{\cos \alpha}$.

In order to prove the validity of the proposed model, the obtained results are compared to the simulation values presented in the previous section. For all the analyzed cases, i.e., for the three wind turbine models and three working frequencies under consideration, the mean error between the simulation values and the values obtained according to Eq. (3) is lower than $0.85 \mathrm{~dB}$. An example to demonstrate that simulation and modeling values are very well aligned is shown in Fig. 8.

\subsection{Scattering from the blades}

From the results of simulations of the RCS patterns, it is clearly shown that the scattering from the blades is significantly lower than the scattering from the mast. Moreover, it should be considered that, as shown in Figs. 5 and 6, the scattering from the blades is strongly dependent on the position of the rotor with respect to the radar. In order to analyze a potential impact situation, therefore, a detailed representation of the blades and all the possible movements of the wind turbine should be needed. However, obtaining detailed representations of actual wind turbine blades is quite difficult, as the blade design is property of the wind turbine manufacturer, and the analysis of hundreds of different combinations of rotor orientation and blades position requires a huge amount of time and effort.

Therefore, instead of obtaining a complete scattering model for the blades, a simpler approach to this issue is characterizing the maximum value of the scattering from the blades. To do so, the maximum RCS value due to the blades for each wind turbine model will be obtained. In fact, as commented before and shown in Fig. 5, the maximum RCS due to the blades corresponds to the contribution of a single blade in vertical position.

From the set of simulations carried out in this analysis, the maximum RCS values from the mast and blades are shown in Table 2. As shown in Sect. 4 when comparing Figs. 2, 3 and 4, these maximum RCS values are frequency dependent. However, if the relation between the maximum RCS from the mast and the maximum RCS from the blades is obtained, it can be observed that this relation remains almost constant for the different frequency bands.

Although their complex geometry prevents us from obtaining simple RCS models to characterize the scattering from the blades, the relation between the maximum RCS from the mast and the maximum RCS from the blades must be proportional to their corresponding dimensions, as the RCS of an object generally depends upon its physical size when its orientation relative to the LoS to the radar is such that a significant area of the object is illuminated (Knott, 2006; Skolnik, 2008).

As a very simple approach, the blade can be represented by a triangle. However, in real blade designs, the profile of the blade rotates from hub toward to the blade tip in order to maintain the angle of attack (Gipe, 2004). Considering this twist angle of the blades, the area of the triangle will be never completely facing the radar. In Spera and Sengupta (1994) it is empirically obtained that the signal scattering efficiency of a blade $\eta$ is dependent on the blade twist according to

$\eta=\exp (-2.30 \Delta \beta)$

where $\Delta \beta$ is the total blade twist from root to tip (rad). This total twist depends on the blade length and design. In commercial wind turbines, total blade twist is typically about $20^{\circ}$. For example, a Vestas V27 model has a total blade twist of $13^{\circ}$ (Gipe, 2004), which provides scattering efficiency values around $0.45-0.60$.

As a rough approach, we will consider a scattering efficiency of $50 \%$ for the wind turbine blade. As later shown in Tables 2 and 3, this assumption leads to a good approximation of the signal scattered by the blades. Therefore, the relative scattering area from the blades $A_{\text {blades }}$ is calculated as

$A_{\text {blades }}=0.5 \frac{w \cdot l}{2}$,

where $w$ is the maximum blade width and $l$ is the blade length.

The mast, by contrast, will be constantly facing the radar with an area that can be approximated by a trapezoid:

$A_{\text {mast }}=\left(r_{1}+r_{2}\right) H$,

where $r_{1}$ and $r_{2}$ are the upper and lower radii of the mast, and $H$ is the mast height.

Thus, the relation $\Delta$ in $\mathrm{dB}$ between the relative scattering area of the mast and blades can be obtained as

$\Delta=10 \log _{10}\left(\frac{4 H\left(r_{1}+r_{2}\right)}{w \cdot l}\right)$.

According to the wind turbine characteristics gathered in Table 1, these relations are calculated and shown in Table 3. If 
Table 2. Maximum RCS values from the mast and blades for the wind turbine models selected for the simulations.

\begin{tabular}{|c|c|c|c|c|c|c|c|c|c|}
\hline & \multicolumn{3}{|c|}{ WT model 1} & \multicolumn{3}{|c|}{ WT model 2} & \multicolumn{3}{|c|}{ WT model 3} \\
\hline & $\begin{array}{c}\text { Mast } \\
(\mathrm{dBsm})\end{array}$ & $\begin{array}{c}\text { Blade } \\
(\mathrm{dBsm})\end{array}$ & $\begin{array}{l}\text { Difference } \\
\quad(\mathrm{dB})\end{array}$ & $\begin{array}{c}\text { Mast } \\
(\mathrm{dBsm})\end{array}$ & $\begin{array}{c}\text { Blade } \\
(\mathrm{dBsm})\end{array}$ & $\begin{array}{l}\text { Difference } \\
\quad(\mathrm{dB})\end{array}$ & $\begin{array}{c}\text { Mast } \\
(\mathrm{dBsm})\end{array}$ & $\begin{array}{c}\text { Blade } \\
\text { (dBsm) }\end{array}$ & $\begin{array}{l}\text { Difference } \\
\quad(\mathrm{dB})\end{array}$ \\
\hline $2.80 \mathrm{GHz}$ & 55.97 & 45.92 & 10.05 & 61.38 & 46.81 & 14.57 & 64.00 & 48.81 & 15.19 \\
\hline $5.65 \mathrm{GHz}$ & 59.95 & 49.42 & 10.53 & 64.32 & 49.74 & 14.58 & 67.03 & 52.10 & 14.93 \\
\hline $9.4 \mathrm{GHz}$ & 62.42 & 51.61 & 10.81 & 66.45 & 52.00 & 14.45 & 69.14 & 54.22 & 14.92 \\
\hline
\end{tabular}

Table 3. Relation $\Delta$ between the relative scattering area of the mast and blades for the wind turbine models selected for the simulations.

\begin{tabular}{lll}
\hline WT model 1 & WT model 2 & WT model 3 \\
\hline $9.90 \mathrm{~dB}$ & $12.65 \mathrm{~dB}$ & $13.38 \mathrm{~dB}$ \\
\hline
\end{tabular}

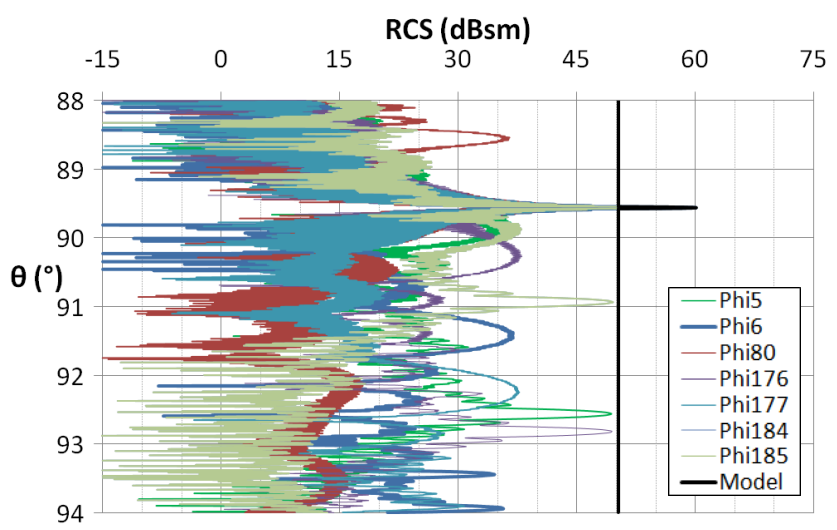

Figure 9. Vertical sections of RCS patterns $\left(\phi=5^{\circ}, 6,80,176\right.$, 177,184 and $185^{\circ}$ ) for wind turbine model 1 (Frequency $5.65 \mathrm{GHz}$, Rotor position P000) and result of the proposed model (black line).

values in Tables 2 and 3 are compared, it can be stated that the relation in $\mathrm{dB}$ between the relative scattering area of the mast and blades can be considered a good approximation of the difference in $\mathrm{dB}$ between the maximum RCS from the mast and the maximum RCS from the blades. Taking this into account, the maximum RCS from the blades (dBsm) can be obtained as

$$
\begin{aligned}
& \sigma_{\text {blades }}=\max \left\{\sigma_{\text {mast }}\right\}-\Delta=10 \log _{10}\left(\frac{2 \pi}{\lambda} r L^{2}\right) \\
& -10 \log _{10}\left(\frac{4 H\left(r_{1}+r_{2}\right)}{w \cdot l}\right) .
\end{aligned}
$$

A comparison of the maximum RCS of the blades from PO simulations and the maximum RCS values calculated according to Eq. (10) is shown in Table 4. As shown in the table, the difference between the values provided by the simulations and the values calculated according to the proposed model are lower than $2 \mathrm{~dB}$ for all the analyzed cases.

\subsection{Converting RCS values to WTC reflectivity values}

In order to model wind turbine clutter, the RCS of a wind turbine must be converted to the equivalent radar reflectivity factor.

The weather radar equation, for distributed targets such as rain, is given by

$P_{\mathrm{r}}=\frac{P_{\mathrm{t}} G^{2} \theta_{0} \phi_{0} c \tau \pi^{3}|K|^{2} z}{1024 \ln (2) \lambda^{2} R^{2}}$,

where $P_{\mathrm{r}}$ is the power received back by radar, $P_{\mathrm{t}}$ is the power transmitted by radar, $\theta_{0}$ and $\phi_{0}$ are the elevation and azimuth beamwidths, $c$ is the speed of light, $\tau$ is the radar pulse length, $|K|^{2}$ is the complex index of refraction of the hydrometeor, $\lambda$ is the wavelength of the radar pulse, $R$ is the distance to the target and $z$ is the radar reflectivity factor (ITU-R, 2009; Rinehart, 1997; Norin and Haase, 2012). The radar reflectivity factor $z$, normally expressed in decibels of reflectivity (dBZ), is the quantity that is used to obtain the rain rate:

$z=\frac{P_{\mathrm{r}} 1024 \ln (2) \lambda^{2} R^{2}}{P_{\mathrm{t}} G^{2} \theta_{0} \phi_{0} c \tau \pi^{3}|K|^{2}}$.

The radar equation for a point target, such as distant wind turbine contained within a range resolution cell, is given by

$P_{\mathrm{r}}=\frac{P_{\mathrm{t}} G^{2} \lambda^{2} \sigma}{64 \pi^{3} R^{4}}$

where $\sigma$ is the RCS of the wind turbine (Knott, 2006).

Assuming that the wind turbine is entirely included within the beam cell resolution of the weather radar, we can compare Eqs. (11) and (13) and then obtain the radar reflectivity factor as

$z=C_{1} \frac{\sigma}{R^{2}}$

where $C_{1}$ is a constant that depends on the parameters of the radar system:

$C_{1}=\frac{16 \ln (2)}{\pi^{6} c} \cdot \frac{\lambda^{4}}{\theta_{0} \phi_{0} \tau} \cdot \frac{1}{|K|^{2}}$. 
Table 4. Comparison of the maximum RCS of the blades from PO simulations and the maximum RCS values calculated according to the proposed model. The third column shows the difference in $\mathrm{dB}$ between the values obtained in the simulations and the values calculated according to the proposed model.

\begin{tabular}{lccccccccc}
\hline & \multicolumn{3}{c}{ WT model 1 } & \multicolumn{3}{c}{ WT model 2 } & \multicolumn{3}{c}{ WT model 3 } \\
\cline { 2 - 10 } & $\begin{array}{c}\text { Simulation } \\
(\mathrm{dBsm})\end{array}$ & $\begin{array}{c}\text { Model } \\
(\mathrm{dBsm})\end{array}$ & $\begin{array}{c}\text { Difference } \\
(\mathrm{dB})\end{array}$ & $\begin{array}{c}\text { Simulation } \\
(\mathrm{dBsm})\end{array}$ & $\begin{array}{c}\text { Model } \\
(\mathrm{dBsm})\end{array}$ & $\begin{array}{c}\text { Difference } \\
(\mathrm{dB})\end{array}$ & $\begin{array}{c}\text { Simulation } \\
(\mathrm{dBsm})\end{array}$ & $\begin{array}{c}\text { Model } \\
(\mathrm{dBsm})\end{array}$ & $\begin{array}{c}\text { Difference } \\
(\mathrm{dB})\end{array}$ \\
\hline $2.80 \mathrm{GHz}$ & 45.92 & 46.08 & 0.16 & 46.81 & 48.72 & 1.91 & 48.81 & 50.62 & 1.82 \\
$5.65 \mathrm{GHz}$ & 49.42 & 50.05 & 0.63 & 49.74 & 51.67 & 1.93 & 52.10 & 53.65 & 1.55 \\
$9.4 \mathrm{GHz}$ & 51.61 & 52.52 & 0.91 & 52.00 & 53.80 & 1.79 & 54.22 & 55.77 & 1.55 \\
\hline
\end{tabular}

\subsection{Complete model for estimating WTC reflectivity in weather radar bands}

Results obtained in the previous subsections are the basis of the complete model to characterize the signal scattering from wind turbines in the weather radar bands proposed in this paper. The proposed simplified model for estimating WTC reflectivity in weather radar bands is summarized in Table 5 .

First, based on the specific characteristics of the wind turbine and the working frequency, the RCS pattern of the mast near the direction normal to the slant surface is obtained. The RCS from the mast is used to determine the main lobe of the RCS pattern of the whole wind turbine.

Then, the maximum RCS value from the blades is calculated, as the maximum RCS value of the mast minus the relation in $\mathrm{dB}$ between the relative scattering areas of the mast and blades. This maximum RCS value from the blades establishes an upper bound, in such a way that all the possible orientations of the nacelle and blades are considered.

In order to combine both patterns and obtain the simplified RCS pattern of the whole wind turbine, the RCS values from the mast are used for angles $\theta$ near the incidence normal to the slanted surface of the mast, i.e., for $\theta$ values such that $\sigma_{\text {mast }} \geq \sigma_{\text {blades. }}$. This way, the main lobe of the RCS pattern of the whole wind turbine is estimated. For incidence angles off the main lobe due to the mast, and up to the limiting angles $\theta$ due to the illumination characteristics of weather radars, the maximum RCS value from the blades is applied.

An example of the results of this proposed RCS model is shown in Fig. 9, together with the simulated results of the RCS pattern for different rotor orientations. In the figure, it can be seen that the maximum RCS of the mast is well approximated by the model, and the mask established off the main lobe covers the scattering from the blades for different rotor orientations.

Once the RCS pattern is completed for a specific illumination condition and configuration of the radar, the estimation of the RCS of the wind turbine is obtained.

Finally, assuming that the whole wind turbine is included within the beam cell resolution of the radar, the corresponding reflectivity value is calculated as described in Table 5 .

\section{Conclusions}

In order to estimate the potential impact of a wind farm on a weather radar service, one of the main issues to be analyzed is wind turbine clutter reflectivity, which is directly related to the radar cross section of wind turbines.

A preliminary study about possible interference problems is the most appropriate way to proceed in order to make the coexistence of wind energy and meteorological services possible. To do so, an estimation of the RCS of the wind turbines to be installed is a must. Although it is possible to obtain RCS values by conventional methods such as MoM and FDTD, they require detailed representations of the wind turbines' design and complex calculations, which are too time consuming and difficult to obtain. On the contrary, typical values that do not take into account the particular features of the case under analysis may lead to significant errors in the impact analysis.

In this paper, the RCS patterns of wind turbines for the weather radar working frequencies have been analyzed. From the obtained results, it can be concluded that the mast is the main scatterer of the wind turbine, featuring a very directive lobe in the direction perpendicular to the slanted surface of the mast. The blades, by contrast, contribute to the total RCS of the wind turbine with secondary lobes that depend on the rotor orientation with respect to the illumination direction and the blades' position.

Based on the above-mentioned conclusions, a simple RCS model to characterize backscattering from wind turbines in the weather radar bands has been proposed. This model takes the RCS from the mast as a reference to estimate the maximum value of the RCS pattern of the whole wind turbine and then calculates the maximum RCS from the blades taking into account the actual dimensions of the wind turbine model. Finally, and assuming that the whole wind turbine is included within the beam cell resolution of the radar, the WTC reflectivity can be directly obtained.

The proposed RCS model can be used to estimate the maximum clutter due to the presence of a wind turbine, estimating the scattered power from the mast. However, even if the Doppler radar under study uses a clutter filter that suppresses stationary objects, the rotating blades of a wind turbine might 
Table 5. Simplified model for estimating WTC reflectivity in weather radar bands.

\begin{tabular}{l}
\hline Model for calculating wind turbine clutter reflectivity \\
\hline 1. Wind turbine RCS \\
$\sigma_{\text {mast }}=10 \log _{10}\left(\frac{2 \pi}{\lambda} r L^{2} \sin (\theta+\alpha)\left(\frac{\sin \left(\frac{2 \pi}{\lambda} L \cos (\theta+\alpha)\right)}{\frac{2 \pi}{\lambda} L \cos (\theta+\alpha)}\right)\right)(\mathrm{dBsm}) \quad$ for $\left.\theta\right|_{\sigma_{\text {mast }} \geq \sigma_{\text {blades }}}$ \\
$\sigma_{\text {blades }}=10 \log _{10}\left(\frac{2 \pi}{\lambda} r L^{2}\right)-10 \log _{10}\left(\frac{4 H\left(r_{1}+r_{2}\right)}{w \cdot l}\right)(\mathrm{dBsm}) \quad$ for $\left.\theta\right|_{\sigma_{\text {mast }}<\sigma_{\text {blades }}}$ \\
Where: $\alpha=\arctan _{\left(\frac{r_{2}-r_{1}}{H}\right) \text { and } L=\frac{H}{\cos \alpha}}$ \\
\hline 2. Wind turbine clutter reflectivity \\
$z=\frac{16 \ln (2)}{\pi^{6} c} \cdot \frac{\lambda^{4}}{\theta_{0} \phi_{0} \tau} \cdot \frac{\sigma}{|K|^{2} R^{2}}$, where $\sigma$ is the RCS in linear values $\left(\mathrm{m}^{2}\right)$ \\
\hline
\end{tabular}

still be detected. As proved in Norin (2015), weather information from radar cells affected by a wind turbine is not always lost. In fact, when precipitation gives rise to reflectivity values stronger than those due to wind turbines, radar data could still be used. Therefore, the reflectivity model proposed in this paper is of interest not only to assess a potential detrimental impact on the performance of a weather radar but also to evaluate to which extent this degradation might exist if reflectivity values from precipitation and wind turbine blades are compared.

This simple WTC reflectivity model aims at being implemented in software planning tools and is expected to make the preliminary impact studies of wind farms on weather radar services easier.

Acknowledgements. A special note of thanks to José Miguel Gutiérrez (AEMet, Spanish Weather Agency) and Lars Norin (SMHI, Swedish Weather Agency), who provided insight and expertise from the perspective of a weather operator.

This work has been partially supported by the Spanish Ministry of Economy and Competitiveness (Ministerio de Economía y Competitividad, project TEC2012-32370) and the University of the Basque Country (Euskal Herriko Unibertsitatea, program for the specialization of the postdoctoral researcher staff).

Edited by: G. Vulpiani

\section{References}

Angulo, I., de La Vega, D., Rodríguez, O., Grande, O., Guerra, D., and Angueira, P.: Analysis of the Mast Contribution to the Scattering Pattern of Wind Turbines in the UHF Band, 5th European Conference on Antennas and Propagation (EuCAP), 2011.

Angulo, I., Montalban, J., Canizo, J., Wu, Y., de la Vega, D., Guerra, D., Angueira, P., and Arrinda, A.: A measurement-based multipath channel model for signal propagation in presence of wind farms in the UHF band, IEEE T. Commun., 61, 4788-4798, 2013.

Angulo, I., de la Vega, D., Cascón, I., Cañizo, J., Wu, Y., Guerra, D., and Angueira, P.: Impact analysis of wind farms on telecommunication services. Renewable and Sustainable Energy Reviews, Volume 32, April 2014, Pages 84-99, ISSN 1364-0321, 2014.

Belmonte, A. and Fabregas, X.: Analysis of Wind Turbines Blockage on Doppler Weather Radar Beams, IEEE Antennas and Wireless Propagation Letters, 9, 670-673, 2010.

Gallardo-Hernando, B., Muñoz-Ferreras, J. M., Pérez-Martínez, F., and Aguado-Encabo, F.: Wind Turbine Clutter Observations and Theoretical Validation for Meteorological Radar Applications, Radar, Sonar \& Navigation, IET, 5, 111-117, Feb. 2011.

Gipe, P.: Wind Power: Renewable Energy for Home, Farm, and Business, 2nd edition, Chelsea Green Publishing, April 1, 2004.

Grande, O., Cañizo, J., Angulo, I., Jenn, D., Danoon, L. R., Guerra, D., and de la Vega, D.: Simplified formulae for the estimation of offshore wind turbines clutter on marine radars, The Scientific World Journal, 982508, doi:10.1155/2014/982508, 2014.

Grande, O., Angulo, I., Jenn, D., Aguado, F., Guerra, D., and de la Vega, D.: Analysis of Wind Turbines Radar Cross Section for Analyzing the Potential Impact on Weather Radars, 2015 9th European Conference on Antennas and Propagation (EUCAP), 1217 April 2015.

IEEE Std 211-1997: IEEE Standard Definitions of Terms For Radio Wave Propagation, 1997.

Isom, B. M., Palmer, R. D., Secrest, G. S., Rhoton, R. D., Saxion, D., Allmon, T. L. , Reed, J., Crum, T., Vogt, R., Detailed Observations of Wind Turbine Clutter with Scanning Weather Radars, J. Atmos. Ocean. Techn., 26, 894-910, 2008.

ITU-R (International Telecommunication Union): Technical and Operational Aspects of Ground-Based Meteorological Radars, Recommendation ITU-RM.1849; International Telecommunication Union, Geneva, Switzerland, 2009.

ITU-R and WMO (International Telecommunication Union and World Meteorological Organization): Handbook, Use of Radio Spectrum for Meteorology: Weather, Water and Climate Monitoring and Prediction, 2008. 
Jenn, D. C.: Radar and Laser Cross Section Engineering, 2nd Edn., Education Series, American Institute of Aeronautics and Astronautics, USA, 2005.

Knott, E. F.: Radar Cross Section Measurements, SciTech Publishing, 2006.

Lemmon, J. J, Carroll, J. E., Sanders, F. H., and Turner, D.: Assessment of the Effects of Wind Turbines on Air Traffic Control radars, NTIA Technical Report TR-08-454, 2008.

Norin, L. and Haase, G.: Doppler Weather Radars and Wind Turbines, Doppler Radar Observations - Weather Radar, Wind Profiler, Ionospheric Radar, and Other Advanced Applications, edited by: Bech, J., InTech, doi:10.5772/39029, available at: http://www.intechopen.com/books/doppler-radarobservations-weather-radar-wind-profiler-ionospheric-radarand-other-advanced-applications/doppler-weather-radars-andwind-turbines (last access: 2 February 2015), 2012.

Norin, L.: A quantitative analysis of the impact of wind turbines on operational Doppler weather radar data, Atmos. Meas. Tech., 8, 593-609, doi:10.5194/amt-8-593-2015, 2015.

Rinehart, R. E.: RADAR for Meteorologists, 3rd edn., Rinehart Pub, 1997.

Siegel, K. M., Alperin, H. A., Bonkowski, R. R., Crispin, J. W., Maffett, A. L., Schensted, C. E., and Schensted, I. V.: Bistatic radar cross sections of surfaces of revolution, J. Appl. Phys., 26, 297 pp., 1955.

Skolnik, M.: Radar Handbook, 3rd Edn., Mc-Graw-Hill, New York, 2008.

Spera, D. A. and Sengupta, D. L.: Equations for Estimating the Strength of TV Signals Scattered by Wind Turbines, NASA Contractor Report 194468, May 1994.
Tristant, P.: Impact of Wind Turbines on Weather Radars Band, World Meteorological Organization, CBS/SG-RFC 2006/Doc. 3.1, 2006.

Van Lil, E., Trappeniers, D., De Bleser, J., and Van de Capelle, A.: Computations of radar returns of wind turbines, in: Proceedings of the 3rd European Conference on Antennas and Propagation (EUCAP), Berlin, Germany, 23-27 March 2009, 38523856, 2009.

Van Lil, E., Trappeniers, D., De Bleser, J., and Van de Capelle, A.: Computation of false echo zones and shadowing for aeronautical and weather radars, in: Proceedings of the 4th European Conference on Antennas and Propagation (EUCAP), Barcelona, Spain, 12 April 2010, 1-5, 2010.

Vogt, R. J., Crum, T. D., Greenwood, W., Ciardi, E. J., and Guenther, R. G.: Recent Efforts to Improve Estimates of and Mitigation of Wind Turbine Clutter Impacts on the WSR-88D, Preprints, 27th Int. Conf. on Interactive Information Processing Systems (IIPS) for Meteorology, Oceanography, and Hydrology, Seattle, WA, Amer. Meteor. Soc., Paper 3A.1, 2011.

WMO (World Meteorological Organization): Impact of Wind Turbines on Weather Radars Band, CBS/SG-RFC 2006/Doc. 3.1(6), 2005.

WMO (World Meteorological Organization): Commission for Instruments and Methods of Observation - Fifteenth Session, WMO-No. 1064, Helsinki, 2-8 September, 2010.

WMO Radar Database: WMO Radar Database, available at: http: //wrd.mgm.gov.tr/ (last access: 15 December 2014), 2014. 